\section{Research Square}

Preprints are preliminary reports that have not undergone peer review.

They should not be considered conclusive, used to inform clinical practice, or referenced by the media as validated information.

\title{
Molecular Epidemiology of Community-Onset Clostridioides Difficile Infections At A Tertiary Hospital In Mainland China: A Ten-Year (2010-2019) Retrospective Study
}

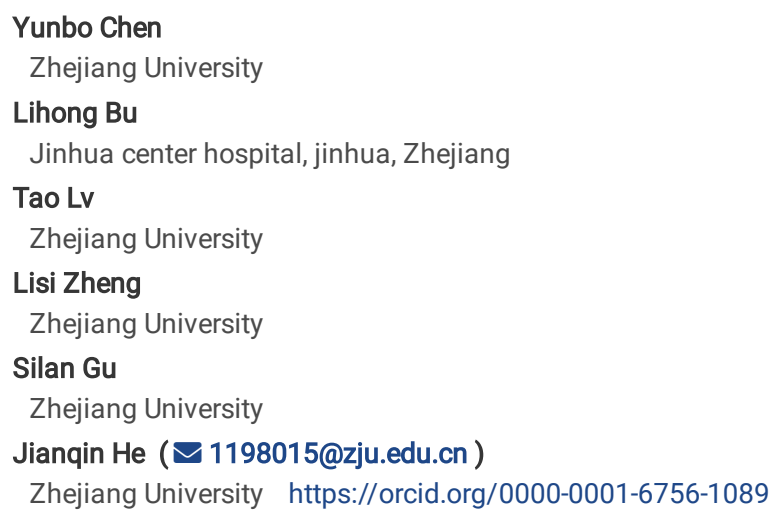




\section{Abstract}

Background: Clostridioides difficile infection (CDI) is an increasingly common disease in healthcare facilities and community settings. However, there are limited reports of community-onset CDI (CO-CDI) in China. We retrospectively analyzed the molecular epidemiology of CO-CDI at a tertiary hospital over a period of 10 years. A total of 1307 stool samples from 1213 outpatients were tested by culturing. The presence of toxin genes ( $t c d A$, $t c d B$, $c d t A$, and $c d t B$ ) were confirmed by PCR. Toxigenic strains were typed using multilocus sequence typing (MLST). Susceptibility to 9 antimicrobials was evaluated using the Etest.

Results: Eighty-nine of 1213 outpatients (7.3\%) had CO-CDI, 4 of these patients (4.5\%) had one or more recurrence, and there were 95 strains of toxigenic $C$. difficile. Among these strains, $82(86.3 \%)$ had the $t c d A$ and $t c d B$ genes $(A+B+)$ and 5 of these 82 strains were positive for the binary toxin genes ( $c d t A$ and $c d t B$ ); the other 13 strains (13.7\%) had the $t c d B$ gene only (A-B+). There were 15 different STs, and the most prevalent were ST-54 (23.2\%), ST-35 (16.8\%), and ST-2 (13.7\%). All strains were susceptible to metronidazole and vancomycin, and had low resistance to moxifloxacin and tetracycline, but had high resistance to ciprofloxacin, clindamycin, and erythromycin. Twenty-three isolates (24.2\%) were multidrug-resistant.

Conclusions: Outpatients with CDI were common during this period in our hospital. The $C$. difficile isolates had high genetic diversity. All isolates were susceptible to metronidazole and vancomycin, and nearly one quarter of all isolates had multidrug resistance.

\section{Background}

Clostridioides difficile is an anaerobic Gram-positive, spore-forming, toxin-producing bacillus that is able to colonize and proliferate in the human gut following changes in the indigenous colonic microbiota. The numbers of $C$. difficile may increase greatly following antibiotic administration, and this can lead to diarrhea, colitis, and pseudomembranous colitis, a condition known as a $C$. difficile infection (CDI)(1). Two structurally similar toxins, toxin A (TcdA, an enterotoxin) and toxin $B(T c d B$, a cytotoxin), are the major virulence factors associated with $\mathrm{CDI}(2)$. However, some toxigenic strains, such as $C$. difficile $\mathrm{BI} / \mathrm{NAP1/RT027}$ (which has caused large epidemics across the developed world) can also produce a third toxin, $C$. difficile binary toxin (CDT), and these strains are associated with increased morbidity and mortality (3).

Most studies of the trends and molecular epidemiology of CDI focused on hospitalized patients. The major and well-established risk factors for hospital-onset $\mathrm{CDI}(\mathrm{HO}-\mathrm{CDI})$ are excessive or non-standard use of antibiotics, older age, prior and prolonged hospitalization, and multiple comorbidities (4). However, CDI is no longer an exclusively nosocomial infection, and recent studies have reported the onset of symptoms in the community (5). In fact, community-onset CDI (CO-CDI) accounts for nearly half of all incident cases and it is now considered a major cause of community-onset diarrhea (6). Moreover, the epidemiology of $\mathrm{HO}-\mathrm{CDI}$ and $\mathrm{CO}-\mathrm{CDI}$ are related, given the frequent movement of patients between hospitals and the community (7). Studies in general populations have compared the characteristics of patients with CO-CDI and HO-CDI and reported that those with CO-CDI were younger and had milder disease. However, some CO-CDI cases still require hospitalization and these individuals may lack the classic health care facility (HCF) risk factors for CDI (8). Because CO-CDI may account for a considerable reservoir of CDI, prevention efforts should also include interventions that reduce CO-CDI(9).

Studies of the molecular epidemiology of CDI in China have mostly examined hospitalized patients, although a few studies have described CO-CDI. We initiated this study to retrospectively analyze the molecular epidemiology of CO-CDI at a tertiary hospital over a period of about 10 years to identify the epidemic strains of $C$. difficile, compare the results with other epidemiological data, and provide an overview of CDI in a single region of China.

\section{Methods}

\section{Collection of $\mathrm{C}$. difficile isolates}

This retrospective study was performed at The First Affiliated Hospital, School of Medicine, Zhejiang University, a 2500-bed tertiary teaching hospital in Hangzhou, Zhejiang, China. Stool samples were collected from outpatients with diarrhea and submitted to the clinical microbiology laboratory between 1 January 2010 and 31 August 2019. Only samples requested for $C$. difficile assay by clinicians were included in the analysis. Patient demographic characteristics, including age, sex, and clinical department of admission, were recorded.

Stool specimens were treated with alcohol to a final concentration of $75 \%$ for spore selection before anaerobic isolation and culturing of $C$. difficile using the selective medium cycloserine-cefoxitin-taurocholate agar (CCFA-TA; Oxoid) supplemented with $7 \%$ sheep blood at $35^{\circ} \mathrm{C}$ for $48 \mathrm{~h}$. Then, the $\mathrm{C}$. difficile isolates were confirmed by matrix-assisted laser desorption ionization time of flight mass spectrometry (MALDI-TOF MS) analysis using the Bruker Daltonics Microflex LT system (Bruker Daltonik GmbH, Bremen, Germany).

\section{Definitions}

A case of CDI was defined as the presence of diarrhea ( $\geq 3$ loose stools/day) with a positive toxin-producing $C$. difficile in the stool. CO-CDI was defined as CDI with onset of symptoms in the community, and did not include patients who first developed symptoms after admission(9). Recurrent CDI was defined as a CDI that was diagnosed within 8 weeks of a prior episode; relapse with the same strain or another strain of $C$. difficile were both designated as recurrent $\mathrm{CDI}(24)$.

\section{Detection of toxin genes by PCR}

Bacteria obtained after growth for $48 \mathrm{~h}$ in anaerobic blood agar culture were suspended in $1 \mathrm{~mL}$ of distilled water in a microcentrifuge tube. Bacterial genomic DNA was then extracted using the simplified alkaline lysis method. All isolated strains were tested for $t c d A$, $t c d B$, and the binary toxin genes $(c d t A$ and $c d t B)$ using the polymerase chain reaction (PCR), as previously described $(10,20)$. 


\section{Multilocus sequence typing (MLST) and analysis}

MLST was used to genotype all toxigenic isolates based on seven housekeeping genes (adk, atpA, $d x r, \operatorname{gly} A, \operatorname{rec} A$, $\operatorname{sod} A$, and $t p i)$, as previously $\operatorname{described}(25)$. Allele designations were obtained from the $C$. difficile PubMLST batch profile query page (http://pubmlst.org/cdifficile/) to obtain the STs.

\section{Antibiotic susceptibility testing}

The antibiotic susceptibility of each toxigenic isolate was analyzed using E-test strips (bioMérieux, Marcy-l'Étoile, France), as previously described(10). Each minimum inhibitory concentration (MIC) was read at the point at which the zone of complete inhibition intersected with the MIC scale, using Clinical and Laboratory Standards Institute (CLSI) guidelines. The selected resistance breakpoints included $8 \mathrm{mg} / \mathrm{L}$ for erythromycin, clindamycin, tetracycline, and the fluoroquinolones, and $32 \mathrm{mg} / \mathrm{L}$ for metronidazole, according to the CLSI interpretative categories approved for anaerobic bacteria. The resistance breakpoints determined by The European Committee on Antimicrobial Susceptibility Testing (EUCAST) were used for vancomycin ( $>2 \mathrm{mg} / \mathrm{L}$ ), linezolid $(>4 \mathrm{mg} / \mathrm{L})$, and rifampicin (> $32 \mathrm{mg} / \mathrm{L}$ ) (http://www.eucast.org/clinical_breakpoints/). The control isolate was C. difficile ATCC 700057.

\section{Data Analysis}

SPSS version 23.0 for Windows (SPSS) was used for statistical analysis.

\section{Ethical approval}

Participants or their legally authorized representatives gave informed consent to be included in the study. Ethical approval for the study was granted by the First Affiliated Hospital, School of Medicine, Zhejiang University (Approval number 1554-1).

\section{Results}

\section{Patient characteristics}

We analyzed 1307 samples from 1213 patients at our institution (median age: 48 years, $47.4 \%$ males) and diagnosed CDI in 89 patients ( $7.3 \%$, median age: 56 years, $60.1 \%$ males) from 1 January 2010 and 31 August 2019 (Table 1). The number of CDI episodes per total tests performed was $49 / 507$ for the clinical gastroenterology department, $7 / 232$ for the emergency department, $8 / 117$ for the clinical infectious disease department, 10/71 for the clinical hematology department, and 15/286 for other departments. Thus, the highest positive rates of CDI were in the clinical hematology department (13.2\%) and the clinical gastroenterology department (9.4\%). Among the 89 positive patients, $3(3.4 \%)$ had a single recurrence and 1 (1.1\%) had multiple recurrences (total recurrence incidence: $4.5 \%$ ). The median age of the 4 patients with recurrences was 53 years, and 3 of them were males.

Table 1

Demographics of outpatient study participants.

\begin{tabular}{|c|c|c|c|}
\hline Characteristic & All outpatients (\%) & Patients without CDI (\%) & Patients with CDI (\%) \\
\hline Total (n) & 1213 & 1124 & 89 \\
\hline \multicolumn{4}{|l|}{ Gender (n) } \\
\hline Male & $575(47.4)$ & $521(46.4)$ & $54(60.7)$ \\
\hline Female & $638(52.6)$ & $603(53.6)$ & $35(39.3)$ \\
\hline \multicolumn{4}{|l|}{ Clinical Department (n) } \\
\hline CIDD & $117(9.6)$ & $109(9.7)$ & $8(9.0)$ \\
\hline ED & $232(19.1)$ & $225(20.0)$ & $7(7.9)$ \\
\hline CGD & $507(41.8)$ & $458(40.7)$ & $49(55.1)$ \\
\hline $\mathrm{CHD}$ & $71(5.9)$ & $61(5.4)$ & $10(11.2)$ \\
\hline ODs & $286(23.6)$ & $271(24.1)$ & $15(16.9)$ \\
\hline Median age, years (range) (year) & $48(2-93)$ & $47(2-93)$ & $56(2-93)$ \\
\hline Male & $49(2-93)$ & $47(2-93)$ & $56(19-93)$ \\
\hline Female & $5548(2-92)$ & $47(2-91)$ & $55(2-92)$ \\
\hline Visiting CIDD & $51(11-93)$ & $50(11-93)$ & $62(32-92)$ \\
\hline Visiting ED & $55(14-92)$ & $54(14-92)$ & $65(53-86)$ \\
\hline Visiting CGD & $54(2-93)$ & $53(2-87)$ & $62(2-93)$ \\
\hline Visiting CHD & $36(2-84)$ & $36(2-84)$ & $38(16-51)$ \\
\hline
\end{tabular}


Analysis of all outpatients with diarrhea indicated the median age was significantly greater in those with than without CDI (56 vs. 47 years, $p=0.004)$. Moreover, stratifying the data by age classes indicated an increased percentage of patients with diarrhea due to $C$. difficile as age increased (Fig. 1). However, there were no differences according to gender.

\section{Molecular epidemiology of the isolates}

We isolated $126(9.6 \%)$ strains from 1307 stool samples, and identified 75.4\% (95/126) toxigenic strains and 24.6\% (31/126) non-toxigenic strains. Among the 95 toxigenic strains, $82(86.3 \%)$ were positive for $t c d A$ and $t c d B$ genes $(A+B+)$ and $13(13.7 \%)$ contained only the $t c d B$ gene $(A-B+)$. Five of the $A+B+$ isolates $(5.3 \%, 5 / 95)$ were positive for $c d t A$ and $c d t B$.

We used MLST to analyze the toxigenic strains, and divided them into 15 different STs. The most prevalent STs were ST-54 (23.2\%, 22/95), ST-35 (16.8\%, $16 / 95)$, ST-2 $(13.7 \%, 13 / 95)$, ST-3 $(9.5 \%, 9 / 95)$, and ST-37 (9/95). We also identified four ST-5 isolates that were positive for $t c d A$, $t c d B$, and the binary toxin genes; two were from the clinical gastroenterology department, one was from the clinical hematology department, and one was from another clinical department.

ST-54 $(22.2 \%, 12 / 54)$, ST-2 $(16.7 \%, 9 / 54)$, ST-3 $(11.1 \%, 6 / 54)$ were the most common in the clinical gastroenterology department, whereas ST-54 $(45.5 \%, 5 / 11)$ and ST-37 $(27.3 \%, 3 / 11)$ were the most common in the clinical hematology department. Among the 4 patients with recurrence, 2 had relapses with the same STs (ST-54 and ST-285), 1 was re-infected another ST (ST-5 then ST-35), 1 was infected 4 times with different STs (ST-2, ST-54, ST-102, and then ST-54).

\section{Antimicrobial resistance}

Our analysis of the distribution of the MICs of the different antimicrobial agents (Table 2) indicated that metronidazole and vancomycin had in vitro activity against all of the toxigenic isolates within a narrow range. Two ST-37 isolates had resistance to linezolid, with MICs above $4 \mu \mathrm{g} / \mathrm{mL}$. Most isolates were resistant to clindamycin (69.4\%) and erythromycin (62.1\%). Analysis of the quinolones indicated $100 \%$ resistance to ciprofloxacin and $22.1 \%$ resistance to moxifloxacin. The resistance to tetracycline was $14.7 \%$ and the resistance to rifampicin was $7.4 \%$. Four of the 7 strains resistant to rifampicin were ST-37. The isolates with binary toxin genes had lower MICs to all tested antibiotics and were all susceptible to erythromycin and moxifloxacin.

Table 2

Resistance of the 95 toxigenic $C$. diffiicle isolates to different antibiotics.

\begin{tabular}{|c|c|c|c|c|c|c|c|c|c|c|}
\hline $\begin{array}{l}\text { ST } \\
\text { (No.of } \\
\text { isolates) }\end{array}$ & $\begin{array}{l}\text { Percentage } \\
\text { of } \\
\text { isolates(\%) }\end{array}$ & Vancomycin & Metronidazole & Linezolid & Clindamycin & Rifampicin & Ciprofloxacin & Moxifloxacin & Erythromycin & Tetı \\
\hline ST-2(13) & 13.68 & $0(0)$ & $0(0)$ & $0(0)$ & $7(53.8)$ & $1(7.7)$ & $7(100)$ & $2(15.4)$ & $3(23.1)$ & $1(7$. \\
\hline ST-3(9) & 9.47 & $0(0)$ & $0(0)$ & $0(0)$ & $8(88.9)$ & $0(0)$ & $9(100)$ & $4(44.4)$ & $4(44.4)$ & $1(1$. \\
\hline ST-5(4) & 4.21 & $0(0)$ & $0(0)$ & $0(0)$ & $1(25)$ & $0(0)$ & $4(100)$ & $0(0)$ & $0(0)$ & $0(0 ;$ \\
\hline ST-8(4) & 4.21 & $0(0)$ & $0(0)$ & $0(0)$ & $0(0)$ & $0(0)$ & $4(100)$ & $0(0)$ & $0(0)$ & $0(0$, \\
\hline ST-11(1) & 1.05 & $0(0)$ & $0(0)$ & $0(0)$ & $1(100)$ & $0(0)$ & $1(100)$ & $0(0)$ & $0(0)$ & $1(11$ \\
\hline ST-33(3) & 3.16 & $0(0)$ & $0(0)$ & $0(0)$ & 1(33.3) & $0(0)$ & $3(100)$ & $0(0)$ & $0(0)$ & $0(0)$ \\
\hline $\begin{array}{l}\text { ST- } \\
35(16)\end{array}$ & 16.84 & $0(0)$ & $0(0)$ & $0(0)$ & $11(68.8)$ & $1(0.6)$ & 16(100) & $3(18.8)$ & $14(87.5)$ & $8(51$ \\
\hline ST-37(9) & 9.47 & $0(0)$ & $0(0)$ & 2(22.2) & $8(88.9)$ & $4(44.4)$ & $9(100)$ & $5(55.6)$ & $9(100)$ & $2(2:$ \\
\hline ST-53(1) & 1.05 & $0(0)$ & $0(0)$ & $0(0)$ & $1(100)$ & $0(0)$ & $1(100)$ & $0(0)$ & $0(0)$ & $0\left(0^{\prime}\right.$ \\
\hline $\begin{array}{l}\text { ST- } \\
54(22)\end{array}$ & 23.16 & $0(0)$ & $0(0)$ & $0(0)$ & 18(81.8) & $1(4.5)$ & $22(100)$ & $3(13.6)$ & 21(95.5) & $1(4$. \\
\hline ST-81(4) & 4.21 & $0(0)$ & $0(0)$ & $0(0)$ & $4(100)$ & $0(0)$ & $4(100)$ & $3(75)$ & $4(100)$ & $0(0)$ \\
\hline $\begin{array}{l}\text { ST- } \\
102(2)\end{array}$ & 2.11 & $0(0)$ & $0(0)$ & $0(0)$ & $1(50)$ & $0(0)$ & $22(100)$ & $0(0)$ & $1(50)$ & $0(0)$ \\
\hline $\begin{array}{l}\text { ST- } \\
129(3)\end{array}$ & 3.16 & $0(0)$ & $0(0)$ & $0(0)$ & $3(100)$ & $0(0)$ & $3(100)$ & $0(0)$ & $3(100)$ & $0(0)$ \\
\hline $\begin{array}{l}\text { ST- } \\
139(2)\end{array}$ & 2.11 & $0(0)$ & $0(0)$ & $0(0)$ & $0(0)$ & $0(0)$ & $2(100)$ & $0(0)$ & $0(0)$ & $0(0 ;$ \\
\hline $\begin{array}{l}\text { ST- } \\
285(2)\end{array}$ & 2.11 & $0(0)$ & $0(0)$ & $0(0)$ & $0(0)$ & $0(0)$ & $2(100)$ & $0(0)$ & $0(0)$ & $0\left(0^{\prime}\right.$, \\
\hline $\begin{array}{l}\text { Total } \\
\text { number } \\
\text { (95) }\end{array}$ & 100 & $0(0)$ & $0(0)$ & $2(2.2)$ & $66(69.4)$ & $7(7.4)$ & $95(100)$ & $21(22.1)$ & $59(62.1)$ & 14( \\
\hline
\end{tabular}

Analysis of multidrug-resistant strains, defined as those with resistance to at least 3 antibiotics, indicated that 24.2\% (23/95) of the toxigenic isolates were multidrug-resistant. These multidrug-resistant strains were ST-35 (7/23), ST-37 (6/23), ST-81 (3/23), ST-3 (3/23), ST-2 (2/23), and ST-54 (2/23). 


\section{Discussion}

Our 10-year surveillance of CO-CDI at a tertiary hospital in China indicated that 89 patients (7.3\%) had CDI and $4.5 \%$ of these patients experienced at least one recurrence. This observation is line with our earlier study, which identified $8.9 \%$ of toxigenic $C$. difficile isolates from inpatients (10). There were two reasons for the small number of CO-CDI patients in this study. Unlike other studies of CO-CDI, which recruited patients admitted within $48 \mathrm{~h}$, we only analyzed individuals who visited outpatient clinics, so it is likely that most patients with severe or difficult-to-treat CO-CDI were admitted to a hospital. Another reason for our smaller number of CO-CDI cases may be the poor awareness of CDI among general healthcare workers, which could have led to missed cases. In the study by Bauer et al., there was no specific request to test for $C$. difficile, which could have caused six of ten cases to be missed (11).

Our outpatients with CDI were older than those without CDI (56 vs. 47 years). However, the median age of our CDI patients was similar to that of inpatients with CDI in our previous study (56 years)(12). A previous study reported that patients with CO-CDI were younger than those with HO-CDI(5). However, the present study clearly showed that CDI patients tended to be older, in that nearly half of the cases (43/89) were 60 or more years-old. Moreover, the median age of the 10 patients from the clinical hematology department was 38 years, and this biased the total median age. Epidemiological studies in Western countries showed that CDI was more common in females than males(13), and a large study of 113 laboratories across England also reported that $67 \%$ of CO-CDI cases were in females $(13,14)$. However, males accounted for more than half of the outpatients in the present study, similar to the proportion of inpatients in our previous study(12). This difference may be due to gender differences in care-seeking behaviors among different countries.

More than half of our cases were from the clinical gastroenterology department, indicating that diarrhea or gastrointestinal discomfort were the most common reasons for visiting the clinic. Patients visiting this department were older than those who visited other outpatient departments. This is consistent with the interpretation that older individuals are more susceptible to $\mathrm{CDI}(15)$. Another interesting finding of the present study is that the positive rate for $\mathrm{CDI}$ was highest in our clinical hematology department, and the median patient age was youngest in this department. Our review of the records of these patients indicated that most of them were follow-up patients who received chemotherapy or haematopoietic stem cell transplants (HSCTs), and these cases of $C$. difficile were healthcare-facility acquired CO-CDI. There is evidence that patients receiving chemotherapy for haematological malignancies or HSCTs have an increased risk for $\mathrm{CDI}(16)$. Thus, it is important for clinicians to consider testing for $C$. difficile in outpatients who develop diarrhea and are older than 60 years or immunocompromised. Recurrences of $\mathrm{CDI}$ are serious, and the management of these patients is challenging. A meta-analysis found that the recurrence rate of CDI was 13 to $50 \%$ among all patients after an initial episode(17). The recurrence in the present study was $4.5 \%$, similar to that reported by Tsai et al. (4.7\%) (18), but lower than reported in another study (15.9\%)(19). We speculate this may be because our CO-CDI patients were not as severely ill as hospitalized patients and because some of our patients were lost to follow-up due to visiting other hospitals.

The $A+B+$ strains were the most common types in the present study, similar to our previous study(10). The epidemiology of $C$. difficile is region-specific. Thus, CDI cases in Europe are mostly from ribotypes RT002 and RT056(13). In the present study, ST-54, ST-35, and ST-2 were the most common STs, similar to our previous study which identified ST-54, ST-35, and ST-37 as the three most common STs among patients hospitalized with CDI(20). Although ST-2 was not among the major STs isolated from inpatients in our previous studies(20), ST-2 is a major ST of $C$. difficile isolated from patients with community-associated $\mathrm{CDI}$ in many European countries(21). Future studies are needed to compare the differences of ST diversity of $C$. difficile isolates in patients with communityonset and hospital-onset CDI. Interestingly, the distributions of STs differed among our different hospital departments. For example, ST-54 was the most common in the clinical gastrointestinal department and clinical hematology department, but ST-37 was the most common in the clinical infectious disease department. In general, the STs of inpatients and outpatients are similar, and a previous study showed that healthcare facilities and community settings had $79 \%$ of the same RTs(22). This may be because outpatients who were hospitalized were reviewed after discharge, especially in the hematology clinic and the infection clinic. Fortunately, none of the isolates was identified as the hypervirulent ST-1 (BI/NAP1/027), but there was one ST-11 (RT078) isolate from the clinical hematology department.

Vancomycin and metronidazole are the only two antimicrobial agents used to treat $\mathrm{CDI}$ in China. Fortunately, all toxigenic $C$. difficile isolates from our outpatients were sensitive to these two antibiotics and the MICs were low. Compared with our previous study, the isolates from outpatients and hospitalized patients had similar resistance profiles(10). However, we observed a low resistance to tetracycline, slightly lower than reported for hospital-acquired CDI but not significantly different from reports in the Asia-Pacific region(23). These differences in antimicrobial resistance may be attributed to differences in exposure to antibiotics in outpatients and individuals in the community, and to the use of different antibiotics in different regions. Another important finding of the present study is that nearly one-quarter of the isolates were multidrug-resistant, much lower than previously reported for isolates from inpatients(10). Based on this, we speculate that most of the strains we identified were from community, where antibiotic use is much less common.

There were some limitations in this study. Firstly, we only analyzed patients visiting clinics, and this may have reduced the number of CO-CDI for those who were admitted within $48 \mathrm{~h}$. Secondly, we did not classify CDI as community-acquired, or healthcare-acquired. Thirdly, we were missing data regarding antibiotic exposure, comorbidities, and outcomes, and could therefore not identify risk factors associated with CDI. Therefore, further research is needed to address these limitations.

\section{Conclusion}

In conclusion, this retrospective study described community-onset CDI at a tertiary hospital over a period of about 10 years. There were many outpatients with $\mathrm{CDI}$, the $C$. difficile isolates had high genetic diversity, and ST-54 was the most common ST. All isolates were susceptible to metronidazole and vancomycin, the resistance rates varied for the other tested antibiotics, and nearly one-quarter of the isolates were multidrug-resistant. Physicians should consider CDI during the differential diagnosis of a patient who presents with community-onset diarrhea or abdominal pain, either alone or in combination. Further studies that analyze whole-genome sequences and epidemiological data are required to investigate the genetic relationships of isolates from hospitalized patients and outpatients, especially follow-up patients. 


\section{List Of Abbreviations}

Clostridioides difficile infection (CDI); community-onset CDI (CO-CDI); hospital-onset CDI (HO-CDI); C. difficile binary toxin (CDT); cycloserine-cefoxitintaurocholate agar (CCFA-TA); matrix-assisted laser desorption ionization time of flight mass spectrometry (MALDI-TOF MS); Multilocus sequence typing (MLST); minimum inhibitory concentration (MIC).

\section{Declarations}

\section{Ethics approval and consent to participate}

Participants or their legally authorized representatives gave informed consent to be included in the study. Ethical approval for the study was granted by the First Affiliated Hospital, School of Medicine, Zhejiang University (Approval number 1554-1).

\section{Consent for publication}

Not applicable.

\section{Availability of data and materials}

The datasets used and/or analysed during the current study are available from the corresponding author on reasonable request.

\section{Competing interests}

The authors declare that they have no competing interests.

\section{Funding}

This work was funded by the National Nature Scientific Fund in China (Grants 82073609).

\section{Authors' contributions}

Yunbo Chen analyzed the data and drafted the paper. Lihong Bu and Tao Lv performed the experiments. Lisi Zheng and Silan Gu revised the analyzed data. Jianqin He designed and revised the paper. Yunbo Chen and Lihong Bu contributed equally to this work. All authors contributed and approved the final article.

\section{Acknowledgements}

Not applicable.

\section{References}

1. Rupnik M, Wilcox MH, Gerding DN. Clostridium difficile infection: new developments in epidemiology and pathogenesis. Nat Rev Microbiol. 2009;7(7):526-36.

2. Pothoulakis C. Pathogenesis of Clostridium difficileassociated diarrhoea. Eur J Gastroenterol Hepatol. 1996;8(11):1041-7.

3. McEllistrem MC, Carman RJ, Gerding DN, Genheimer CW, Zheng L. A hospital outbreak of Clostridium difficile disease associated with isolates carrying binary toxin genes. Clin Infect Dis. 2005;40(2):265-72.

4. Bassetti M, Villa G, Pecori D, Arzese A, Wilcox M. Epidemiology, diagnosis and treatment of Clostridium difficile infection. Expert Rev Anti Infect Ther. 2012;10(12):1405-23.

5. Crobach MJT, Notermans DW, Harmanus C, Sanders I, De Greeff SC, Kuijper EJ. Community-Onset Clostridioides Difficile Infection in Hospitalized Patients in The Netherlands. Open Forum Infect Dis. 2019;6(12):ofz501.

6. Guh AY, Mu Y, Winston LG, Johnston H, Olson D, Farley MM, et al. Trends in U.S. Burden of Clostridioides difficile Infection and Outcomes. N Engl J Med. 2020;382(14):1320-30.

7. Tan XQ, Verrall AJ, Jureen R, Riley TV, Collins DA, Lin RT, et al. The emergence of community-onset Clostridium difficile infection in a tertiary hospital in Singapore: a cause for concern. Int J Antimicrob Agents. 2014;43(1):47-51.

8. Kwon SS, Gim JL, Kim MS, Kim H, Choi JY, Yong D, et al. Clinical and molecular characteristics of community-acquired Clostridium difficile infections in comparison with those of hospital-acquired C. difficile. Anaerobe. 2017;48:42-6.

9. Mora Pinzon MC, Buie R, Liou JI, Shirley DK, Evans CT, Ramanathan S, et al. Outcomes of Community and Healthcare-onset Clostridium difficile Infections. Clin Infect Dis. 2019;68(8):1343-50.

10. Chen YB, Gu SL, Shen P, Lv T, Fang YH, Tang LL, et al. Molecular epidemiology and antimicrobial susceptibility of Clostridium difficile isolated from hospitals during a 4-year period in China. J Med Microbiol. 2018;67(1):52-9.

11. Bauer MP, Veenendaal D, Verhoef L, Bloembergen P, van Dissel JT, Kuijper EJ. Clinical and microbiological characteristics of community-onset Clostridium difficile infection in The Netherlands. Clin Microbiol Infect. 2009;15(12):1087-92.

12. Xu Q, Chen Y, Gu S, Lv T, Zheng B, Shen P, et al. Hospital-acquired Clostridium difficile infection in Mainland China: A seven-year (2009-2016) retrospective study in a large university hospital. Sci Rep. 2017;7(1):9645. 
13. Fawley WN, Davies KA, Morris T, Parnell P, Howe R, Wilcox MH. Enhanced surveillance of Clostridium difficile infection occurring outside hospital, England, 2011 to 2013. Euro Surveill. 2016;21(29).

14. Ofori E, Ramai D, Dhawan M, Mustafa F, Gasperino J, Reddy M. Community-acquired Clostridium difficile: epidemiology, ribotype, risk factors, hospital and intensive care unit outcomes, and current and emerging therapies. J Hosp Infect. 2018;99(4):436-42.

15. Donskey CJ. Clostridium difficile in Older Adults. Infect Dis Clin North Am. 2017;31(4):743-56.

16. Gu SL, Chen YB, Lv T, Zhang XW, Wei ZQ, Shen P, et al. Risk factors, outcomes and epidemiology associated with Clostridium difficile infection in patients with haematological malignancies in a tertiary care hospital in China. J Med Microbiol. 2015;64(Pt 3):209-16.

17. Garey KW, Sethi S, Yadav Y, DuPont HL. Meta-analysis to assess risk factors for recurrent Clostridium difficile infection. J Hosp Infect. 2008;70(4):298304.

18. Tsai CS, Hung YP, Lee JC, Lee NY, Chen PL, Syue LS, et al. Community-onset Clostridium difficile infection at a tertiary medical center in southern Taiwan, 2007-2015. J Microbiol Immunol Infect. 2018;51(2):243-50.

19. Visconti V, Brunetti G, Cuomo MR, Giordano A, Raponi G. Nosocomial-acquired and community-onset Clostridium difficile infection at an academic hospital in Italy: Epidemiology, recurrences and toxin genes distribution. J Infect Chemother. 2017;23(11):763-8.

20. Chen YB, Gu SL, Wei ZQ, Shen P, Kong HS, Yang Q, et al. Molecular epidemiology of Clostridium difficile in a tertiary hospital of China. J Med Microbiol. 2014;63(Pt 4):562-9.

21. Bauer MP, Notermans DW, van Benthem BH, Brazier JS, Wilcox MH, Rupnik M, et al. Clostridium difficile infection in Europe: a hospital-based survey. Lancet. 2011;377(9759):63-73.

22. Furuya-Kanamori L, Riley TV, Paterson DL, Foster NF, Huber CA, Hong S, et al. Comparison of Clostridium difficile Ribotypes Circulating in Australian Hospitals and Communities. J Clin Microbiol. 2017;55(1):216-25.

23. Luo Y, Cheong E, Bian Q, Collins DA, Ye J, Shin JH, et al. Different molecular characteristics and antimicrobial resistance profiles of Clostridium difficile in the Asia-Pacific region. Emerg Microbes Infect. 2019;8(1):1553-62.

24. Bauer MP, Kuijper EJ, van Dissel JT. European Society of Clinical Microbiology and Infectious Diseases (ESCMID): treatment guidance document for Clostridium difficile infection (CDI). Clin Microbiol Infect. 2009;15(12):1067-79.

25. Griffiths D, Fawley W, Kachrimanidou M, Bowden R, Crook DW, Fung R, et al. Multilocus sequence typing of Clostridium difficile. J Clin Microbiol. 2010;48(3):770-8.

\section{Figures}

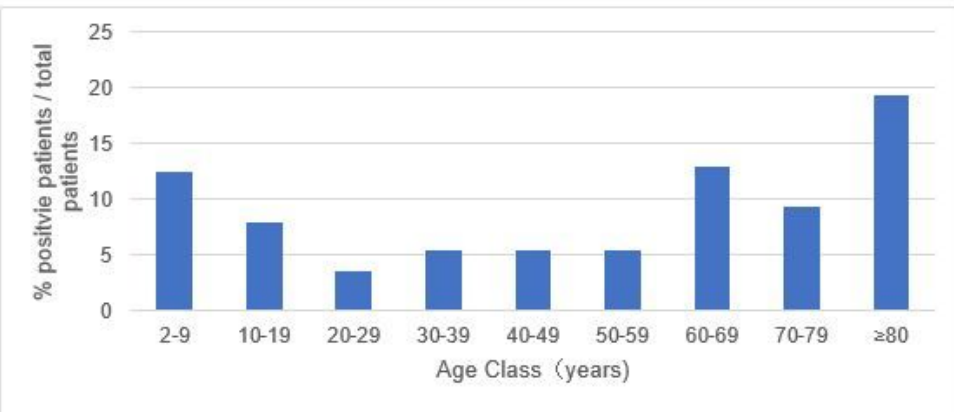

Figure 1

Percentages of outpatients with CDI in different age classes 